\title{
Clinical efficacy and safety of evolocumab for low- density lipoprotein cholesterol reduction
}

\author{
This article was published in the following Dove Press journal: \\ Vascular Health and Risk Management \\ 19 April 2016 \\ Number of times this article has been viewed
}

\author{
Courtney A Henry \\ Ronald A Lyon \\ Hua Ling \\ Department of Pharmacy Practice, \\ School of Pharmacy, Hampton \\ University, Hampton, VA, USA
}

Correspondence: Hua Ling Department of Pharmacy Practice, School of Pharmacy, Hampton University, I00 E Queen Street, Hampton,VA 23668, USA

Email hua.ling@hamptonu.edu

\begin{abstract}
Multiple categories of medications have been developed to manage lipid profiles and reduce the risk of cardiovascular events in patients with heart disease. However, currently marketed medications have not solved the problems associated with preventing and treating cardiovascular diseases completely. A substantial population of patients cannot take advantage of statin therapy due to statin intolerance, heart failure, or kidney hemodialysis, suggesting a need for additional effective agents to reduce low-density lipoprotein cholesterol (LDL-C) levels. Proprotein convertase subtilisin/kexin type 9 (PCSK9) was discovered in 2003 and subsequently emerged as a novel target for LDL-C-lowering therapy. Evolocumab is a fully human monoclonal immunoglobulin G2 (IgG2) directed against human PCSK9. By inactivating PCSK9, evolocumab upregulates LDL receptors causing increased catabolism of LDL-C and the consequent reduction of LDL-C levels in blood. Overall, evolocumab has had notable efficacy, with LDL-C reduction ranging from 53\% to $75 \%$ in monotherapy and combination therapies, and is associated with minor adverse effects. However, studies regarding the ability of evolocumab to reduce mortality as well as long-term safety concerns are limited. The fact that the drug was introduced at a cost much higher than the existing medications and shows a low incremental mortality benefit suggests that many payers will consider evolocumab to have an unfavorable cost-benefit ratio.
\end{abstract}

Keywords: PCSK9, hyperlipidemia, evolocumab, LDL-C, familial hypercholesterolemia

\section{Introduction}

Since the 1960s, the medical community has been looking for ways to prevent (primary prevention) and treat (secondary prevention) cardiovascular disease through management of cholesterol levels. ${ }^{1,2}$ In reducing the risk for coronary heart disease, lowering low-density lipoprotein cholesterol (LDL-C), raising high-density lipoprotein cholesterol (HDL-C), and lowering triglycerides appeared to have the potential for reducing both morbidity and mortality. ${ }^{3}$ Multiple categories of medications have been developed to manage lipid profiles and reduce the risk of cardiovascular events.

Unfortunately, currently marketed medications have not solved the problems associated with preventing and treating cardiovascular disease completely. Fibric acid derivatives and niacin have not consistently demonstrated the ability to reduce mortality in patients at risk for cardiovascular events. ${ }^{4,5}$ The most commonly used medications for lipid disorders, statins, have demonstrated the ability to reduce mortality in a variety of patient populations. ${ }^{6,7}$ However, a substantial population of patients cannot take advantage of statin therapy due to statin intolerance, heart failure, or kidney hemodialysis. ${ }^{8-12}$ The introduction of ezetimibe provided another alternative for patients who 
could not tolerate or otherwise reach their target LDL-C goal using statins. The result of the IMPROVE-IT trial demonstrated a modest benefit of combining ezetimibe with simvastatin. ${ }^{13}$ However, many patients could not reach their target goals using the combination product. ${ }^{14}$ Lomitapide and mipomersen are novel agents approved by the US Food and Drug Administration (FDA) a few years ago and indicated as an adjunct therapy for patients with homozygous familial hypercholesterolemia (HoFH). Ongoing clinical research evaluating other novel investigational agents with unique mechanisms of action includes CETP inhibitors and dual PPAR- $\alpha / \gamma$ agonists. Nevertheless, neither agent has made it to the market yet due to safety or efficacy concerns.

The FDA recently approved two medications, evolocumab (Repatha; Amgen, Thousand Oaks, CA, USA) and alirocumab (Praluent; Sanofi and Regeneron Pharmaceuticals Inc., Tarrytown, NY, USA), within the class of PCSK9 inhibitors. The ninth member of the proprotein convertase family, PCSK9, was discovered in 2003 and subsequently emerged as a novel target for LDL-C lowering therapy..$^{15}$ The circulating LDL-C is usually cleared from the blood via hepatocyte receptor-mediated endocytosis. The LDL-C particles first bind to LDL receptors (LDL-Rs) on the hepatocyte cell membrane followed by internalization into endosome; then, after dissociation from LDL-C at the acidic $\mathrm{pH}$ of the endosome, the receptors are recycled back to the cell surface for another cycle of LDL-C catabolism. ${ }^{16}$ Human PCSK9 plays a critical role in controlling plasma LDL-C by binding to the epidermal growth factor-like domain of the LDL-Rs, and thus facilitating the degradation of receptor within the lysosome. Though the exact mechanism of this process is not entirely known, PCSK9 inhibitors can prevent PCSK9 from degrading the LDL-Rs and significantly increase the expression of LDL-Rs, which further reduces the levels of LDL-C in the plasma. ${ }^{17}$ Mutations of PCSK9 have been reported to significantly impact cardiovascular outcomes. ${ }^{18}$ The development of gain-of-function mutations in PCSK9 is associated with autosomal dominant hypercholesterolemia, while loss-of-function mutations are thought to correlate with lower LDL-C and reduced coronary heart disease without additional deleterious effects. ${ }^{18}$

In this article, we describe the current state of evolocumab and make recommendations regarding its potential status in therapy.

\section{Clinical pharmacology}

Evolocumab is a $141.8 \mathrm{kDa}$, fully human monoclonal immunoglobulin G2 (IgG2) directed against human PCSK9. ${ }^{19}$
Evolocumab strongly binds to PCSK9 and prevents circulating PCSK9 from binding to the LDL-Rs. ${ }^{20}$ The inhibition of PCSK9-mediated LDL-R degradation enables the LDL-Rs to recycle back to the liver cell surface. By inactivating PCSK9, evolocumab upregulates LDL-Rs, resulting in increased catabolism of LDL-C and the consequent reduction of LDL-C levels in the blood. Recently approved by the FDA, evolocumab is indicated as an adjunct to diet and maximally tolerated statin therapy for the treatment of adults with heterozygous familial hypercholesterolemia $(\mathrm{HeFH})$ or clinical atherosclerotic cardiovascular disease, who require additional lowering of LDL-C. ${ }^{21}$ Evolocumab is also approved as an adjunct to diet and other LDL-lowering therapies for the treatment of patients with $\mathrm{HoFH}$ who require additional lowering of LDL-C. ${ }^{21}$

\section{Pharmacokinetics and pharmacodynamics}

The absorption model of evolocumab varies based on the dose. In $140 \mathrm{mg}$ single-dosing regimen, absorption of evolocumab follows nonlinear pharmacokinetics, whereas in $420 \mathrm{mg}$ dosing it follows linear pharmacokinetics. The maximum suppression of circulating unbound PCSK9 was observed by 4 hours after single subcutaneous injection of $140 \mathrm{mg}$ or $420 \mathrm{mg}$ of evolocumab. The absolute bioavailability is estimated to be $\sim 72 \%$ in both the doses time of maximum concentration observed $\left(T_{\max }\right)$ and steady state are achieved in 3-4 days and 12 weeks, respectively. Evolocumab is composed solely of amino acids and carbohydrates; therefore, it is unlikely to be eliminated via hepatic or renal mechanisms. Unlike many statins that are extensively metabolized in both the liver and/ or gut and eliminated primarily in bile, the metabolism and elimination of evolocumab are expected to follow the immunoglobulin clearance pathways, resulting in degradation to small peptides and individual amino acids. When used as an adjunct with statin therapy, it is worthy of note that clearance of evolocumab was observed to increase by $20 \%{ }^{21,22}$ However, no dosage adjustment is recommended with the concurrent usage of statin. Evolocumab at either dose shows no notable differences in effectiveness between subgroups, such as age, race, sex, and body mass index, therefore adjustments for aforementioned factors are not necessary. ${ }^{21} \mathrm{~A}$ comparison of the pharmacokinetics and pharmacodynamics parameters of evolocumab and atorvastatin is given in Table 1.

\section{Role of evolocumab in the treatment of hypercholesterolemia}

The Phase III studies have continued to show affirmation to include evolocumab in LDL-C reduction therapy. Overall, evolocumab has had notable efficacy, with LDL-C reduction 
Table I A comparison of the pharmacokinetics and pharmacodynamics parameters of evolocumab and atorvastatin

\begin{tabular}{|c|c|c|c|}
\hline Parameters & Mean (SD) of I $40 \mathrm{mg}$ evolocumab & Mean (SD) of $420 \mathrm{mg}$ evolocumab ${ }^{\mathrm{a}}$ & Atorvastatin ${ }^{b}$ \\
\hline $\mathrm{C}_{\max }$ mean & $18.6(7.3) \mu \mathrm{g} / \mathrm{mL}$ & $59.0(17.2) \mu g / m L$ & $\mathrm{~N} / \mathrm{A}$ \\
\hline $\mathrm{AUC}_{\text {last }}$ & $188(98.6)$ day $\bullet \mu g / m L$ & $924(346)$ day $\cdot \mu \mathrm{g} / \mathrm{mL}$ & $\mathrm{N} / \mathrm{A}$ \\
\hline Time to reach the steady state & 12 weeks & 12 weeks & $\mathrm{N} / \mathrm{A}$ \\
\hline The steady-state volume of distribution & $3.3 \pm 0.5 \mathrm{~L}$ & $3.3 \pm 0.5 \mathrm{~L}$ & $381 \mathrm{~L}$ \\
\hline Systemic clearance & $12(2) \mathrm{mL} / \mathrm{h}$ & $12(2) \mathrm{mL} / \mathrm{h}$ & $625 \mathrm{~mL} / \mathrm{min}$ \\
\hline The expected half-life & $11-17$ days & $11-17$ days & 14 hours \\
\hline
\end{tabular}

Note: a Data from Repatha ${ }^{\mathrm{TM}}$ (package insert). ${ }^{21}{ }^{\mathrm{b}}$ Data from Lennernas H. Clinical pharmacokinetics of atorvastatin. Clin. Pharmacokinet. 2003;42(I3): I I4I-I I60.43

Abbreviations: SD, standard deviation; $\mathrm{AUC}_{\text {last }}$, the area under the curve up to the last measurable concentration; $\mathrm{C}_{\text {max }}$, maximum concentration observed; $\mathrm{N} / \mathrm{A}$, not applicable.

ranging from $53 \%$ to $75 \%$ in monotherapy and combination therapies, and is associated with minor adverse effects. ${ }^{23-30} \mathrm{The}$ effectiveness of evolocumab on cardiovascular outcomes has not been evaluated in any completed Phase III trials to date, making the clinical value of PCSK9 inhibitors questionable.

\section{Evolocumab as monotherapy}

In the MENDEL-2 study, evolocumab was used as a monotherapy in comparison with ezetimibe and placebo in patients with hypercholesterolemia. ${ }^{23}$ The double-blind trial examined the response to monthly and biweekly subcutaneous injections of evolocumab in comparison to ezetimibe and placebo. The trial randomized 614 patients aged 18-80 years with LDL-C fasting plasma levels of $\geq 100$ and $<190 \mathrm{mg} / \mathrm{dL}$ and Framingham risk scores $\leq 10 \%$. Patients in evolocumab group achieved a reduction in LDL-C of $55 \%-57 \%$ in comparison to placebo, and the reduction was $38 \%-40 \%$ greater to that observed in ezetimibe group $(P<0.001$ for all compared scenarios). Results of monthly and biweekly injections were very similar. Significant reduction in total cholesterol, apolipoprotein B, non-HDL-C, total cholesterol/HDL-C, apolipoprotein $\mathrm{B} /$ apolipoprotein $\mathrm{A}$ 1, and lipoprotein(a) was also observed in evolocumab group compared with both placebo and ezetimibe groups $(P<0.001)$. Adverse events occurred evenly across the study, which resulted in only $2.3 \%$ of the patients using evolocumab to terminate the study versus $3.9 \%$ and $3.2 \%$ on placebo and ezetimibe, respectively. Only two cases of adverse events related to the study were considered serious, which included acute pancreatitis and increased transaminase and creatine kinase levels. The study did not evaluate the reduction in risk of cardiovascular events. ${ }^{23}$

\section{Evolocumab combined with statin}

Evolocumab given in addition to statin therapy is able to significantly reduce LDL-C levels. The LAPLACE-2 study was a double-blind, randomized study to evaluate the safety, tolerability, and efficacy of evolocumab in combination with moderate-to-high intensity statin therapy in patients with hypercholesterolemia and mixed dyslipidemia. ${ }^{24}$ After receiving the statin treatment for 4 weeks, 1,899 patients were randomized to receive evolocumab and placebo, placebo and ezetimibe, or placebo only, in addition to their statin therapies. The results of the study revealed that evolocumab reduced LDL-C by $63 \%-75 \%$ versus placebo (mean of measurement taken at weeks 10 and 12). Patients receiving evolocumab every 2 weeks with high-intensity statin therapy were able to lower their LDL-C levels to $35-38 \mathrm{mg} / \mathrm{dL}$, and similar results were also reported for patients receiving monthly evolocumab treatment with their LDL-C levels decreasing to $33-35 \mathrm{mg} / \mathrm{dL}$. The most common adverse events occurring in evolocumab-treated patients included back pain and headache. One patient had detectable binding antibodies at the end of the trial. Less than $2 \%$ of the patients receiving evolocumab treatment discontinued due to the development of adverse events.

\section{Long-term effectiveness}

In the DESCARTES study, patients with LDL-C greater than $75 \mathrm{mg} / \mathrm{dL}$, triglyceride levels below $400 \mathrm{mg} / \mathrm{dL}$, and aged 18-75 years were grouped based on their risk according to the Adult Treatment Panel III of the National Cholesterol Education Program. ${ }^{25}$ After their risk classification was determined, patients began therapy with diet alone or diet with additional therapy. The additional therapy included atorvastatin monotherapy or atorvastatin with ezetimibe. After pretreatment, the patients who still had an LDL-C greater than $75 \mathrm{mg} / \mathrm{dL}$ were placed randomly to receive either $420 \mathrm{mg}$ evolocumab or placebo monthly for 52 weeks. In comparison to the placebo group, patients receiving evolocumab showed a significant reduction of $57 \%$ in LDL-C levels $(P<0.001)$. There were also improvements in the patients' lipid profiles, which included reduced apolipoprotein B, non-HDL-C, lipoprotein(a), and triglycerides. Common side effects observed in patients treated with evolocumab included nasopharyngitis $(10.5 \%)$, upper respiratory tract infection (9.3\%), influenza (7.5\%), and back pain $(6.2 \%){ }^{25}$ 
One of the most anticipated results from the PCSK9 trials is the ability of this new class of drug to reduce cardiovascular events and associated morbidity and mortality. Long-term studies are providing insight into the outcomes of evolocumab treatment, as seen in the ongoing OSLER Trial (Phase II/III). ${ }^{26}$ In this long-term study, 4,465 patients who had participated in at least one Phase II or Phase III study of evolocumab before were randomized to receive open-label evolocumab $420 \mathrm{mg}$ every month in addition to the standard of care, or the standard of care alone for either another 52 weeks (OSLER) or 156 weeks (OSLER-2). Evolocumab significantly reduced the LDL-C level by $\sim 60 \%$ compared with standard of care, with the mean LDL-C level of $\sim 50 \mathrm{mg} / \mathrm{dL}$ in the evolocumab group during the trial. The most significant aspect of this trial was the evaluation of rate of occurrence of cardiovascular events in patients receiving evolocumab versus patients receiving the standard therapy. After a follow-up period of $\sim 11$ months, the rates of occurrence of cardiovascular events in enrolled patients were $2.18 \%$ for standard of care and $0.95 \%$ for evolocumab, respectively (hazard ratio $0.47 ; 95 \%$ confidence interval [CI]: 0.28-0.78; $P=0.003)$.

\section{Evolocumab for statin intolerance}

To date, there are few effective options to manage lipid disorders for a substantial group of patients with statin intolerance that occurs in $10 \%-15 \%$ of the patients..$^{8}$ In the GAUSS- 2 study, patients aged $18-80$ years, diagnosed with hypercholesterolemia, and documented statin intolerance (of at least two or more statins) were treated to evaluate if LDL-C levels would be reduced by evolocumab or ezetimibe treatment for 12 weeks. ${ }^{27}$ In this double-blind study, 307 patients were randomized to receive oral placebo with evolocumab (140 mg every 2 weeks or $420 \mathrm{mg}$ every month) or oral $10 \mathrm{mg}$ ezetimibe daily with placebo. Evolocumab reduced LDL-C levels by $53 \%-56 \%$ and $37 \%-39 \%$ when compared to ezetimibe and placebo groups, respectively $(P<0.001)$. Abnormal laboratory results and emergency events occurred equally across both the groups, and muscle-related adverse events were most common occurring in $12 \%$ of the patients using evolocumab, and $23 \%$ of the patients treated with ezetimibe. This study revealed a possible future for new treatment options in a population with high cholesterol levels and intolerance to statin therapy.

\section{Familial hypercholesterolemia}

For some of the genetic disease states, traditional LDL-C lowering treatments, including statins, have not been able to achieve optimal cholesterol goals and reduce atheroscle- rotic cardiovascular disease risk. In the RUTHERFORD-2 trial, patients diagnosed with $\mathrm{HeFH}$ and on a stable statin therapy for 4 weeks were randomized in a double-blind study receiving either evolocumab (140 mg every 2 weeks or 420 mg every month) or placebo. ${ }^{28}$ At week $12,63 \%-68 \%$ of the patients achieved the LDL-C value $<70 \mathrm{mg} / \mathrm{dL}$ in the evolocumab group, compared with $2 \%$ of the patients in the placebo group. Over 12 weeks, the mean of LDL-C level in the evolocumab group was reduced by $60 \%$ in comparison to baseline values. The most common adverse events included nasopharyngitis and muscle-related adverse events. No serious events occurred that were considered to be related to the study. ${ }^{28}$

The TESLA Part B study is the only Phase III trial that evaluated the efficacy of evolocumab in patients with HoFH. ${ }^{29}$ Fifty patients who had been on a stable statin therapy for at least 4 weeks were randomized in a 2:1 ratio to receive subcutaneous injections of evolocumab $420 \mathrm{mg}$ or placebo every 4 weeks for 12 weeks. At 12 weeks, the LDL-C level was reduced by $\sim 31 \%$ ( $95 \%$ CI: $18 \%-44 \%$; $P<0.0001)$ compared with placebo. Patients receiving evolocumab did not experience any serious adverse events, but more frequently reported upper respiratory tract infection (9\%) and influenza $(9 \%)$, and elevated levels of liver enzymes were reported twice as often in the evolocumab group. ${ }^{29}$ Note that one single patient with negative LDL-R mutation in both alleles in the evolocumab group experienced a $10 \%$ increase in LDL-C level from baseline, suggesting that the patients with negative LDL-R mutation in both the alleles may not respond to evolocumab therapy.

\section{Ongoing Phase III outcome study}

The FOURIER trial is an active Phase III outcome study that is expected to be completed in February 2018. This study includes patients aged 40-85 years who are diagnosed with cardiovascular disease and are considered to be at high risk for a recurrent cardiovascular event, and is evaluating whether the addition of evolocumab can reduce cardiovascular-related events such as stroke, myocardial infarction, and death. ${ }^{30}$ Additional long-term studies are necessary to determine if evolocumab has the ability to not only reduce LDL-C levels significantly, but also improve the rates of morbidity and mortality.

\section{Safety, tolerability, and immunogenicity of evolocumab}

In general, evolocumab is well tolerated with an overall incidence of adverse events similar to placebo, based on current clinical database. The treatment with evolocumab showed 
adverse events comparable with placebo and alternative therapies and obtained high completion rates of $96 \%-99 \%$ for four Phase III trials (12 weeks), and a 92\% completion rate for a long-term study versus $89 \%$ for standard of care. ${ }^{23-30}$ There were no major safety concerns reported in the clinical trials. Similar incidences of common adverse events, such as allergic reactions, neurocognitive events, and musculoskeletal events, were observed and are presented in Table 2. It would be expected that evolocumab would be well tolerated based on the completion rates and adverse events rate in the clinical trials and the comparability of the number of adverse reactions with control groups.

Injection-site reactions could potentially negatively affect patient adherence. The incidence of injection-site reactions were $3.2 \%$ and $3 \%$ in evolocumab and placebo groups, respectively. ${ }^{21}$ The most common injection site reactions were erythema, pain, and bruising. Two Phase III trials evaluating the patients' ability to properly administer evolocumab with either an autoinjector or prefilled syringe have been completed, though no results have been published currently. ${ }^{31,32}$ Results of these Phase III studies may further examine patient tolerability and adherence to the subcutaneous route of administration.

A significant number of patients in clinical trials had at least one LDL-C level less than $25 \mathrm{mg} / \mathrm{dL}$; however, the long-term effects of very low LDL-C levels and adverse events induced by evolocumab are unknown in these trials. ${ }^{26}$ Therefore, more data and long-term studies regarding the safety of achieving very low levels of LDL-C, induced by evolocumab, are needed. Furthermore, a recent study also found that the Friedewald equation used in most clinical trials underestimates LDL-C levels in patients with very low LDL-C levels. ${ }^{33}$ The lack of reliability of LDL-C estimation is particularly worrisome given that the efficacy of evolocumab in lowering LDL-C in most Phase III trials could be exaggerated to some extent.

Evolocumab, as a protein, theoretically has the ability to trigger an immune response. Unwanted immunogenicity

Table 2 Adverse events of evolocumab

\begin{tabular}{lll}
\hline Adverse events & $\begin{array}{l}\text { Evolocumab group } \\
\text { (\%) }\end{array}$ & $\begin{array}{l}\text { Control group } \\
\text { (\%) }\end{array}$ \\
\hline Allergic reactions & 3.2 & 3.0 \\
Neurocognitive events & 0.6 & 0.2 \\
Musculoskeletal events & 14.3 & 12.8 \\
Nasopharyngitis & 5.9 & 4.8 \\
Upper respiratory tract & 3.2 & 2.7 \\
$\quad$ infection & & \\
Back pain & 3.0 & 2.7 \\
Nausea & 2.1 & 1.8 \\
\hline
\end{tabular}

can produce antidrug antibodies inactivating the biological agents and diminishing the therapeutic effects. For example, a positive rate of $61 \%$ was observed for antidrug antibodies to infliximab, a chimeric antibody, with decreased clinical response. ${ }^{34}$ Evolocumab is a fully humanized monoclonal antibody with significantly less immunogenicity compared with the chimeric antibody. ${ }^{35}$ The immunogenicity of evolocumab has been evaluated, and results showed that the presence of antidrug binding antibodies is unlikely to impact the efficacy and safety of evolocumab. Only $0.1 \%$ of the patients treated with evolocumab tested positive for the development of binding antibodies, and none of them tested positive for neutralizing antibodies. ${ }^{21}$ The long-term effects of evolocumab in the presence of antidrug binding antibodies are unknown.

\section{Discussion}

Given the consistent effects of evolocumab on LDL-C reduction, as reported in numerous clinical scenarios, the inhibition of PCSK9 appears to be a promising approach to reduce the risk for cardiovascular disease. While we are waiting for the results of the Phase III FOURIER trial, the debate about whether evolocumab-induced LDL-C reduction is sufficient to be translated into reduction in cardiovascular disease is heated after the FDA approval of evolocumab. In a broader sense, should LDL-C still be considered as the best surrogate for cardiovascular disease, especially considering the residual cardiovascular risk despite maximum LDL-C reduction? In the 2013 American College of Cardiology and the American Heart Association cholesterol guidelines, "treat to target" strategy is no longer advocated. ${ }^{36}$ Some new evidence suggests that LDL particles, non-HDL-C, the apolipoprotein B/apolipoprotein A1 ratio, and lipoprotein(a) may be better surrogate markers for cardiovascular mortality than LDL-C in future. ${ }^{33,37-39}$ Nevertheless, the recently published IMPROVE-IT study provides the strongest clinical trial evidence supporting the concept of LDL-C reduction as a surrogate for prevention of long-term cardiovascular outcomes in high-risk patients with acute coronary syndromes. In the IMPROVE-IT study, a linear relationship between the reductions in LDL-C and the proportionate reductions in the incidence of major cardiovascular events was observed, confirming the LDL-C hypothesis which is "the lower the better". ${ }^{13}$ With the strong evidence from the IMPROVE-IT study, there is no doubt that evolocumab-induced LDL-C reduction will lead to a greater reduction in cardiovascular events in the FOURIER trial.

Although we are optimistic about the efficacy of evolocumab, the current data from clinical trials are inadequate to characterize the long-term safety of evolocumab, which raises 
some concern. To date, there is only one completed doubleblinded 52-week trial, and the duration of many other trials is only 12 weeks. Further research is needed to characterize the long-term safety and rule out unwanted off-target effects of evolocumab to assure the widespread use.

The introduction of evolocumab into the market could have the potential to result in a major change in the current clinical practices. As seen in the clinical trials, evolocumab could be a choice for patients with familial hypercholesterolemia and others at high risk of cardiovascular disease, with high levels of LDL-C despite optimal statin therapy or having statin intolerance. Besides, the cost of evolocumab should be an important factor in interpreting "high risk" and determining the appropriate candidates.

The Institute for Clinical and Economic Review developed a draft report estimating the comparative value of the PCSK9 inhibitors. ${ }^{40}$ The report estimated cost per quality adjusted life year (QALY) modeled over 20 years. For familial hypercholesterolemia, it estimates an incremental value of \$681,000/QALY when adding PCSK9-inhibitors to the treatment. For secondary prevention in patients with a prior history of cardiovascular disease and intolerant to statins, it estimates an incremental value of $\$ 506,000 / \mathrm{QALY}$ when adding PCSK9 inhibitors. The Institute for Clinical and Economic Review also estimated that the price of evolocumab would need to decrease from $\$ 14,300$ to $\$ 2,412$ per year $(-83 \%)$ in order to achieve a target of $\$ 50,000 /$ QALY. A similar evaluation estimated an approximate \$5,000/QALY when using atorvastatin for secondary prevention. ${ }^{41}$

Most of the trials used for the analysis lasted only 6 months. Extrapolating these results over 20 years poses the risk of significantly under- or overestimating the benefits and harms of these medications. Using the meta-analysis conducted by Navarese et al, we can estimate the incremental value of adding PCSK9 inhibitors to therapy for 6 months. ${ }^{42}$ On a weighted basis, these medications reduced absolute allcause mortality by $0.22 \%$ ( $0.31 \%$ PCSK 9 inhibitor-treated versus $0.53 \%$ comparator) (odds ratio [OR]: $0.45,95 \% \mathrm{CI}$ : $0.23-0.86, P=0.015)$. With a 6-month cost of $\$ 7,150$ for evolocumab the estimated cost to reduce one death would be $\sim \$ 3,250,000$.

The relatively low value and lack of long-term results suggest that payers will attempt to limit the evolocumab treatment to a very select group of patients that meet strict criteria for use. In addition, the two currently marketed PCSK9 inhibitors demonstrated similar results. This provides an opportunity for payers to negotiate better prices, similar to what occurred with the new medications for hepatitis $\mathrm{C}$.

\section{Conclusion}

In summary, evolocumab has demonstrated high potency for lowering LDL-C levels and side effects comparable to placebo. Though the efficacy in cardiovascular outcomes is still unclear, the current available evidence supports its use in patients with $\mathrm{HeFH}$ or clinical atherosclerotic cardiovascular disease, who require additional lowering of LDL-C. Evolocumab can also be used in HoFH patients with an LDL-R gene defective in at least one of the two affected alleles. In general, evolocumab is relatively safe compared to placebo, either as monotherapy or combined with ezetimibe or statins. However, to date, there is limited evidence addressing its long-term safety concerns, which need further clinical study. The fact that the drug is introduced at a cost much higher than the existing medications and has a low incremental mortality benefit suggests that many payers will consider evolocumab to have an unfavorable cost-benefit ratio. Thus, it will be very necessary for prescribers to carefully select the target patients and manage the cost effectively.

\section{Disclosure}

The authors report no conflicts of interest in this work.

\section{References}

1. Braunstein JB, Cheng A, Cohn G, Aggarwal M, Nass CM, Blumenthal RS. Lipid disorders: justification of methods and goals of treatment. Chest. 2001;120(3):979-988.

2. Senders A, Sando K, Wahbeh H, Hiller AP, Shinto L. Managing psychological stress in the multiple sclerosis medical visit: Patient perspectives and unmet needs. J. Health Psychol. Epub 2014 Dec 19.

3. National Cholesterol Education Program Expert Panel on Detection, Evaluation, and Treatment of High Blood Cholesterol in Adults (Adult Treatment Panel III). Third report of the National Cholesterol Education Program (NCEP) Expert Panel on Detection, Evaluation, and Treatment of High Blood Cholesterol in Adults (Adult Treatment Panel III) final report. Circulation. 2002;106(25):3143-3421.

4. Investigators A-H, Boden WE, Probstfield JL, et al. Niacin in patients with low HDL cholesterol levels receiving intensive statin therapy. N Engl J Med. 2011;365(24):2255-2267.

5. Group AS, Ginsberg HN, Elam MB, et al. Effects of combination lipid therapy in type 2 diabetes mellitus. $N$ Engl J Med. 2010;362(17):1563-1574.

6. Taylor F, Huffman MD, Macedo AF, et al. Statins for the primary prevention of cardiovascular disease. Cochrane Database Syst Rev. 2013;1:CD004816.

7. Cholesterol Treatment Trialists' (CTT) Collaborators, Mihaylova B, Emberson J, et al. The effects of lowering LDL cholesterol with statin therapy in people at low risk of vascular disease: meta-analysis of individual data from 27 randomised trials. Lancet. 2012;380(9841):581-590.

8. Joy TR, Hegele RA. Narrative review: statin-related myopathy. Ann. Int. Med. 2009;150(12):858-868.

9. Fellstrom BC, Jardine AG, Schmieder RE, et al. Rosuvastatin and cardiovascular events in patients undergoing hemodialysis. $N$ Engl $J$ Med. 2009;360(14):1395-1407.

10. Kjekshus J, Apetrei E, Barrios V, et al. Rosuvastatin in older patients with systolic heart failure. N Engl J Med. 2007;357(22):2248-2261.

11. Gissi HFI, Tavazzi L, Maggioni AP, et al. Effect of rosuvastatin in patients with chronic heart failure (the GISSI-HF trial): a randomised, doubleblind, placebo-controlled trial. Lancet. 2008;372(9645):1231-1239. 
12. Wanner C, Krane V, Marz W, et al. Atorvastatin in patients with type 2 diabetes mellitus undergoing hemodialysis. $N$ Engl $\mathrm{J} \mathrm{Med}$. 2005;353(3):238-248.

13. Cannon CP, Blazing MA, Giugliano RP, et al. Ezetimibe added to statin therapy after acute coronary syndromes. $N \mathrm{Engl} \mathrm{J} \mathrm{Med}$. 2015;372(25):2387-2397.

14. Bohula EA, Giugliano RP, Cannon CP, et al. Achievement of dual lowdensity lipoprotein cholesterol and high-sensitivity C-reactive protein targets more frequent with the addition of ezetimibe to simvastatin and associated with better outcomes in IMPROVE-IT. Circulation. 2015;132(13):1224-1233.

15. Cohen JC, Boerwinkle E, Mosley TH, Jr, Hobbs HH. Sequence variations in PCSK9, low LDL, and protection against coronary heart disease. N Engl J Med. 2006;354(12):1264-1272.

16. Horton JD, Cohen JC, Hobbs HH. Molecular biology of PCSK9: its role in LDL metabolism. Trends Biochem Sci. 2007;32(2):71-77.

17. Stein EA, Mellis S, Yancopoulos GD, et al. Effect of a monoclonal antibody to PCSK9 on LDL cholesterol. N Engl J Med. 2012;366(12): 1108-1118.

18. Abifadel M, Varret M, Rabes JP, et al. Mutations in PCSK9 cause autosomal dominant hypercholesterolemia. Nat Genet. 2003; 34(2):154-156.

19. Cicero AF, Colletti A, Borghi C. Profile of evolocumab and its potential in the treatment of hyperlipidemia. Drug Des Devel Ther. 2015;9:3073-3082.

20. Stein E, Wasserman S, Dias C, Scott R, Raal F. AMG145. Drug Future. 2013;38:451-459.

21. Repatha ${ }^{\mathrm{TM}}$ [package insert]. Thousand Oaks, CA: Amgen Inc.,; 2015.

22. Gazzerro P, Proto MC, Gangemi G, et al. Pharmacological actions of statins: a critical appraisal in the management of cancer. Pharmacol. Rev. 2012;64(1):102-146.

23. Koren MJ, Lundqvist P, Bolognese M, et al. Anti-PCSK 9 monotherapy for hypercholesterolemia: the MENDEL-2 randomized, controlled phase III clinical trial of evolocumab. J. Am. Coll. Cardiol. 2014;63(23):2531-2540.

24. Robinson JG, Nedergaard BS, Rogers WJ, et al. Effect of evolocumab or ezetimibe added to moderate- or high-intensity statin therapy on LDL-C lowering in patients with hypercholesterolemia: the LAPLACE-2 randomized clinical trial. JAMA. 2014;311(18):1870-1882.

25. Blom DJ, Hala T, Bolognese M, et al. A 52-week placebo-controlled trial of evolocumab in hyperlipidemia. N Engl J Med. 2014;370(19): 1809-1819.

26. Sabatine MS, Giugliano RP, Wiviott SD, et al. Efficacy and safety of evolocumab in reducing lipids and cardiovascular events. N Engl JMed. 2015;372(16):1500-1509.

27. Stroes E, Colquhoun D, Sullivan D, et al. Anti-PCSK9 antibody effectively lowers cholesterol in patients with statin intolerance: the GAUSS-2 randomized, placebo-controlled phase 3 clinical trial of evolocumab. J. Am. Coll. Cardiol. 2014;63(23):2541-2548.

28. Raal FJ, Stein EA, Dufour R, et al. PCSK9 inhibition with evolocumab (AMG 145) in heterozygous familial hypercholesterolaemia (RUTHERFORD-2): a randomised, double-blind, placebo-controlled trial. Lancet. 2014;385(9965):331-340.

29. Raal FJ, Honarpour N, Blom DJ, et al. Inhibition of PCSK9 with evolocumab in homozygous familial hypercholesterolaemia (TESLA Part B): a randomised, double-blind, placebo-controlled trial. Lancet. 2015;385(9965):341-350.

Vascular Health and Risk Management

\section{Publish your work in this journal}

Vascular Health and Risk Management is an international, peerreviewed journal of therapeutics and risk management, focusing on concise rapid reporting of clinical studies on the processes involved in the maintenance of vascular health; the monitoring, prevention and treatment of vascular disease and its sequelae; and the involvement of
30. Amgen. Further cardiovascular outcomes research with PCSK9 inhibition in subjects with elevated risk (FOURIER). 2015. Available from: https:/clinicaltrials.gov/ct2/show/NCT01764633?term=FOURIER\&r ank=6. Accessed August 25, 2015.

31. Amgen. Study to Assess in Home Use of Evolocumab (AMG 145) Administration Using Either an Automated Mini-doser or a Prefilled Autoinjector/Pen. 2013. Available from: https://clinicaltrials.gov/ct2/ show/NCT01879319?term=20120356\&rank=1. Accessed August 25, 2015.

32. Amgen. Study to Assess In-home Use of Evolocumab (AMG 145) Using a Prefilled Syringe or a Prefilled Autoinjector/Pen. 2013. Available from: https://clinicaltrials.gov/show/NCT01849497. Accessed August 25, 2015.

33. Walldius G, Jungner I. The apoB/apoA-I ratio: a strong, new risk factor for cardiovascular disease and a target for lipid-lowering therapya review of the evidence. J. Int. Med. 2006;259(5):493-519.

34. Baert F, Noman M, Vermeire S, et al. Influence of immunogenicity on the long-term efficacy of infliximab in Crohn's disease. $N$ Engl J Med. 2003;348(7):601-608

35. Harding FA, Stickler MM, Razo J, DuBridge RB. The immunogenicity of humanized and fully human antibodies: residual immunogenicity resides in the CDR regions. MAbs. 2010;2(3):256-265.

36. Stone NJ, Robinson JG, Lichtenstein AH, et al. 2013 ACC/AHA guideline on the treatment of blood cholesterol to reduce atherosclerotic cardiovascular risk in adults: a report of the American College of Cardiology/American Heart Association Task Force on Practice Guidelines. J Am Coll Cardiol. 2014;63(25 Pt B):2889-2934.

37. Cromwell WC, Otvos JD, Keyes MJ, et al. LDL particle number and risk of future cardiovascular disease in the Framingham offspring study - implications for LDL management. J Clin Lipidol. 2007;1(6):583-592.

38. Emerging Risk Factors Collaboration, Erqou S, Kaptoge S, et al. Lipoprotein(a) concentration and the risk of coronary heart disease, stroke, and nonvascular mortality. JAMA. 2009;302(4):412-423.

39. Boekholdt SM, Arsenault BJ, Mora S, et al. Association of LDL cholesterol, non-HDL cholesterol, and apolipoprotein B levels with risk of cardiovascular events among patients treated with statins: a metaanalysis. JAMA. 2012;307(12):1302-1309.

40. (ICER) TIfCaER. PCSK9 inhibitor therapies for high cholesterol: effectiveness, value, and value-based price benchmarks. 2015. Available from: http://cepac.icer-review.org/wp-content/uploads/2015/04/ PCSK9_Draft_Report_0908151.pdf. Accessed October 9, 2015.

41. Robson J. Lipid modification: cardiovascular risk assessment and the modification of blood lipids for the primary and secondary prevention of cardiovascular disease. Heart. 2008;94(10):1331-1332.

42. Navarese EP, Kolodziejczak M, Schulze V, et al. Effects of proprotein convertase subtilisin/kexin type 9 antibodies in adults with hypercholesterolemia: a systematic review and meta-analysis. Ann. Int. Med. 2015;163(1):40-51.

43. Lennernas H. Clinical pharmacokinetics of atorvastatin. Clin. Pharmacokinet. 2003;42(13):1141-1160.

metabolic disorders, particularly diabetes. This journal is indexed on PubMed Central and MedLine. The manuscript management system is completely online and includes a very quick and fair peer-review system, which is all easy to use. Visit http://www.dovepress.com/ testimonials.php to read real quotes from published authors. 